\title{
REVISÃO SISTEMÁTICA COM METANÁLISE PARA AVALIAÇÃO DA EFETIVIDADE DO TEMOZOLOMIDE ASSOCIADO AO BEVACIZUMAB NO TRATAMENTO DA GLIOBLASTOMA MULTIFORME
}

\author{
A. P. L. OLIVEIRA ${ }^{1}$, M. J. B. M. REGO ${ }^{1}$, M. G. R. PITTA $^{1}$ \\ ${ }^{1}$ Universidade Federal de Pernambuco, Centro de Ciências Biológicas, \\ Departamento de Bioquímica. \\ E-mail para contato: paulalopesoliveira@gmail.com \\ RESUMO: Objetivo: avaliar a efetividade do temozolomide associado ao bevacizumab \\ no tratamento dos pacientes portadores de Glioblastoma Multiforme, uma neoplasia \\ cerebral primária do Sistema Nervoso Central. Metodologia: revisão sistemática com \\ metanálise de ensaios clínicos controlados e randomizados (ECCR) pesquisados nas \\ bases de dados MEDLINE (via Pubmed), Center for Reviews and Dissemination (CRD) \\ e Biblioteca COCHRANE. Os resultados mostraram uma melhora significativamente do \\ Bevacizumab associado à Terapia de Radiação e Temozolomide para os desfechos \\ progressão de sobrevivência e taxa de sobrevivência de 6 meses. Em geral, a adição de \\ bevacizumab ao tratamento com radioterapia-temozolomide pode ser uma estratégia de \\ terapia eficaz para melhorar a sobrevivência de progressão e a taxa de sobrevivência de \\ 6 meses; contudo, outros ensaios clínicos com bom delineamento para verificar eventos \\ adversos dos tratamentos e qualidade de vida dos pacientes devem ser realizados.
}

Palavras-chaves: neoplasia, radiação, Sistema Nervoso Central

ABSTRACT: Objective: to evaluate the effectiveness of temozolamide compared to its association with bevacizumab in the treatment of patients with Glioblastoma Multiforme, a primary cerebral neoplasm of the Central Nervous System. METHODS: Systematic review with a meta-analysis of randomized controlled clinical trials (RCTs) searched in MEDLINE databases (via Pubmed), Center for Reviews and Dissemination (CRD) and COCHRANE Library. The results showed a significant improvement of Bevacizumab associated with Radiation Therapy and Temozolomide for the survival progression outcomes and 6-month survival rate. In general, the addition of bevacizumab to radiotherapy-temozolomide therapy may be an effective therapy strategy to improve survival of progression and survival rate of 6 months; however, other well-designed clinical trials to verify adverse treatment events and patients' quality of life should be performed

Key words: neoplasia, radiation, Central Nervous System

\section{INTRODUÇÃO}

A abordagem terapêutica para o tratamento do Glioblastoma Multiforme (GBM) consiste basicamente na remoção da massa tumoral, seguida de radioterapia concomitante com quimioterapia por temozolomide (TMZ). Dentre outros fármacos para o tratamento da 
doença, o Temozolomide pertence a uma nova classe de agentes alquilantes, que tem baixo peso molecular, sendo $100 \%$ absorvida por via oral. Por ser uma substância lipofílica, atravessa a barreira hemo-encefálica, o que faz alcançar tumores cerebrais. Estudos de fase I e II comprovam que o TMZ, quando aplicado a doses padrões, atinge concentrações intratumorais muito melhores do que muitas terapias citotóxicas. O Bevacizumab, um antiangiogênico, consiste na ruptura dos vasos sanguíneos do tumor. No entanto, apresenta reações adversas, que são minimizadas através da infusão intra-arterial cerebral. Os tumores relacionados ao Sistema Nervoso Central permanecem como um grande desafio ao desenvolvimento de técnicas de diagnóstico e tratamento, de forma que o resultado deste estudo pode indicar um aumento da perspectiva de vida aos pacientes portadores do GBM, quando utilizado o Bevacizumab associado ao TMZ.

\section{MATERIAIS E MÉTODOS}

Foram incluídos ensaios clínicos controlados randomizados (ECCR) e Revisões Sistemáticas (com ou sem meta-análise) de estudos ECCR, cujo delineamento tenha avaliado a eficácia e segurança do temozolomide associado ao bevacizumab. A pesquisa abrangeu uma estratégia de busca predefinida nas bases de dados: MedLine via Pubmed, Center of review and dissemination CRD, LILACS, Biblioteca Cochrane e o registro de ensaios clínicos Clinicaltrials.

Pergunta de pesquisa: "O uso do temozolomide associado ao bevacizumab aumentam a expectativa de vida dos pacientes portadores de glioblastoma multiforme?".

Análise dos dados: calculado o risco relativo (RR) para as variáveis dicotômicas, e a diferença da média (DM) para as variáveis continuas, em ambos os casos, foram considerados um intervalo de confiança (IC) de 95\%. A análise estatística foi realizada utilizando o Review Manager 5.3 (RevMan). Os desfechos avaliados foram quantitativamente resumidos utilizando o método Mantel-Haenszel para as variáveis calculadas pela métrica do RR, e o inverso da variância (IV) para as variáveis calculadas pela métrica foi testada com o $\mathrm{Chi}^{2}$, sendo o $\mathrm{Tau}^{2}$ (método IV) e $\left(\mathrm{I}^{2}\right)$, utilizados para estimar a variância entre os estudos e como medida do nível de heterogeneidade estatística.

\section{RESULTADOS E DISCUSSÃO}

Todos os estudos relevantes foram identificados pela pesquisa inicial da literatura. As principais características dos estudos incluídos são apresentadas na Tabela 1:

Tabela 1- Características dos estudos incluídos na revisão sistemática:

\begin{tabular}{|c|c|c|c|c|c|c|}
\hline Estudo & Tipo de estudo & Tempo de seguin & Quant. amos & População & Intervenção & Comparador \\
\hline $\begin{array}{l}\text { Lai } \mathrm{A}, \\
\text { et. al, } \\
2011\end{array}$ & $\begin{array}{l}\text { Estudo open-label, } \\
\text { prospectivo, } \\
\text { multicêntrico de um } \\
\text { único braço de fase } \\
\text { II. }\end{array}$ & 24,2 meses & 70 vs. 100 & $\begin{array}{l}\text { Pacientes recém- } \\
\text { diagnosticados com } \\
\text { Glioblastoma Multiforme } \\
\text { (GBM). Análise patológica } \\
\text { central (O.E.S, W.H.Y) }\end{array}$ & $\begin{array}{l}\text { Bevacizumab + Terapia } \\
\text { Radiação e Temozolomi }\end{array}$ & $\begin{array}{l}\text { Terapia de } \\
\text { Radiação e } \\
\text { Temozolomide }\end{array}$ \\
\hline $\begin{array}{l}\text { Gilbert M, } \\
2014\end{array}$ & $\begin{array}{l}\text { Estudo randomizado, } \\
\text { cego, controlado por } \\
\text { placebo, teste de fase }\end{array}$ & 20,5 meses & 320 vs. 317 & $\begin{array}{l}\text { Recém-diagnosticados com } \\
\text { gliobastoma Astrocitoma de } \\
\text { IV (OMS). Incluíram tambén } \\
\text { Karnofsky com pelo menos } 7 \\
\text { anos e adequada função renal } \\
\text { hepát }\end{array}$ & $\begin{array}{l}\text { Bevacizumab + Terapia } \\
\text { Radiação e Temozolomi }\end{array}$ & $\begin{array}{l}\text { Terapia de Radiação } \\
\text { Temozolomide }\end{array}$ \\
\hline $\begin{array}{l}\text { Chinot, } \\
\text { O, et al, } \\
2014\end{array}$ & $\begin{array}{l}\text { Estudo randomizado, } \\
\text { cego }\end{array}$ & $\begin{array}{l}12,3 \text { meses para } \\
\text { o grupo } \\
\text { bevacizumab e } \\
8,5 \text { meses para }\end{array}$ & 458 vs. 463 & $\begin{array}{l}\text { Recém-diagnosticados com } \\
\text { gliobastoma Astrocitoma de } \\
\text { IV (OMS). }\end{array}$ & $\begin{array}{l}\text { Bevacizumab + Terapia } \\
\text { Radiação e Temozolomi }\end{array}$ & $\begin{array}{l}\text { Terapia de Radiação } \\
\text { Temozolomide }\end{array}$ \\
\hline
\end{tabular}




\begin{tabular}{|c|l|l|l|l|l|l|}
\hline & & $\begin{array}{l}\text { o grupo } \\
\text { placebo. }\end{array}$ & & & \\
\hline $\begin{array}{c}\text { Balana } \\
\text { C, et. al, } \\
2016\end{array}$ & $\begin{array}{l}\text { Estudo randomizado } \\
\text { de fase II }\end{array}$ & 18 meses & 45 vs. 48 & $\begin{array}{l}\text { Pacientes com } \\
\text { glioblastoma não } \\
\text { resseguido, diagnosticado } \\
\text { por biópsia incluindo } \\
\text { craniotomia estereotáxica } \\
\text { ou aberta, e não tenha } \\
\text { recebido nenhum } \\
\text { tratamento prévio. }\end{array}$ & $\begin{array}{l}\text { Bevacizumab + Terapia } \\
\text { Radiação e Temozolomi }\end{array}$ & $\begin{array}{l}\text { Terapia de Radiação } \\
\text { Temozolomide }\end{array}$ \\
& & & & & \\
\hline
\end{tabular}

A avaliação de risco de viés realizado através da ferramenta Cochrane considera 7 domínios (random sequence generation, allocation concealment, blinding of participants and personnel, blinding of outcome assessment, incomplet outcome data, selective reporting, other bias). Dos 3 estudos incluídos, dois apresentaram baixo risco de viés em todos os domínios 30 , dois estudos apresentaram alto risco de viés para ocultação de alocação 31,33 e um apresentou risco de viés indeterminado para cegamento de avaliadores de desfecho, desfechos incompletos e relato de desfecho seletivo.

A metanálise foi realizada para os desfechos sobrevivência geral, progressão livre de sobrevivência e para taxa de sobrevivência de 6 meses e encontra-se expressas nas figuras 2,3 e 4 .

O desfecho sobrevivência foi avaliada em todos os estudos incluídos na revisão, com presença de heterogeneidade média (I2:66\%) e sem diferença estatística entre os grupos intervenção e controle.

A progressão de sobrevivência também mensurada em todos os artigos foi estatisticamente favorável para grupo intervenção (MD: 0.73 [0.63-0.84], p<0.0001) e apresentou baixa heterogeneidade (I2:36\%).

Foi realizada a comparação da taxa de sobrevivência de 6 meses dos quatro estudos incluídos na revisão. A razão de chances para taxa de sobrevivência de 6 meses foi estatisticativamente favorável para o grupo intervenção (MD: 0.59 [0.36-0.96], $\mathrm{p}=0.04$ ) com heterogeneidade média I2:52.

A presente metanálise incluiu três ensaios clínicos randomizados duplo-cegos de controle de placebo (RCTs) mais um ensaio clínico de um único braço aberto. Os resultados mostraram uma melhora significativamente do Bevacizumab associado à Terapia de Radiação e Temozolomide para os desfechos progressão de sobrevivência e taxa de sobrevivência de 6 meses. O presente estudo corrobora com os achados de Fu et al., 2015, no qual a progressão de sobrevivência apresentou superior para o grupo controle e a taxa de sobrevivência de 6 meses, antes inconclusiva, mostrou-se estatisticamente favorável para o grupo intervenção após a inclusão de Balana et al.,deste modo, aumentando o poder estatístico da amostra. 
Figura 2 -Metanálise para sobrevivência geral:

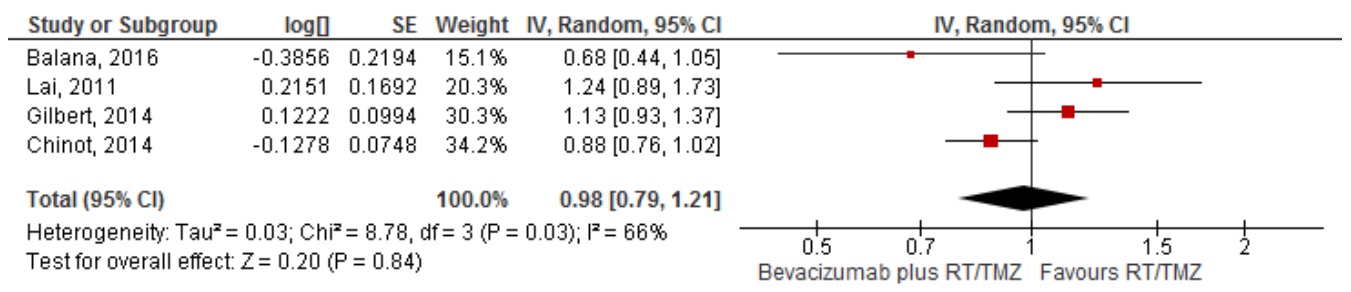

Figura 3 -Metanálise para progressão de sobrevivência

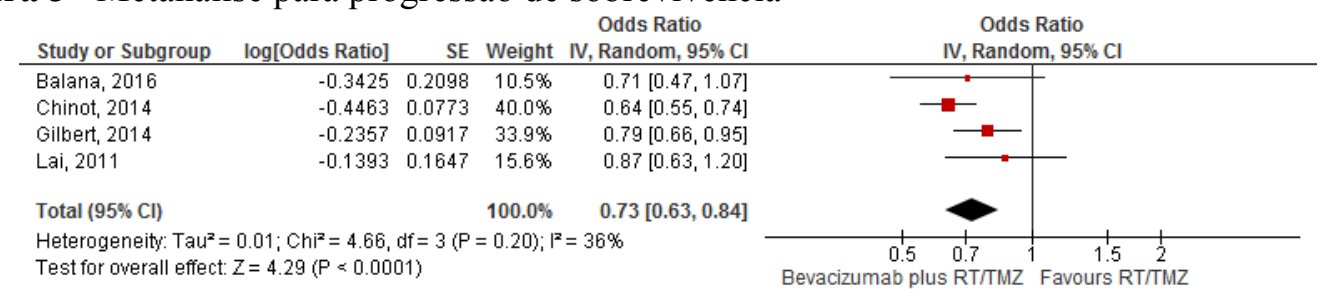

Figura 4 - Forest plot de comparação. Bevacizumab mais radioterapia / temozolomida (RT / TMZ) versus RT / TMZ . Razão de chances para taxa de sobrevivência de 6 meses:

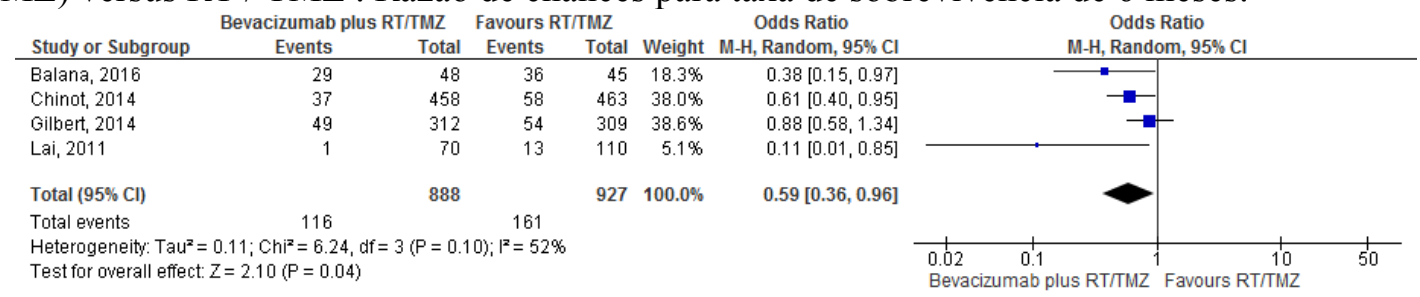

\section{CONCLUSÃO}

Em geral, a adição de bevacizumab ao tratamento com radioterapia-temozolomide pode ser uma estratégia de terapia eficaz para melhorar a sobrevivência de progressão e taxa de sobrevivência de 6 meses; contudo, outros ensaios clínicos com bom delineamento para verificar eventos adversos dos tratamentos e qualidade de vida dos pacientes devem ser realizados.

\section{REFERÊNCIAS}

COHEN MH, JOHNSON JR, PAZDUR R. Food and Drug Administration approval summary: temozolomide plus radiation therapy for the treatment of newly diagnosed glioblastoma multiforme. Clin Cancer Res 2005;11(19):6767-6771.

DEHDASHTI AR, HEGI, REGLI L et al. New trends in medical management of glioblastoma multiforme: the role of temozolomide chemotherapy. Neurosurg Focus 2006;20(4):E6 